\title{
EDITORIAL
}

\section{ERGONOMÍA E INVESTIGACIÓN EN EL SECTOR SALUD}

\author{
ERGONOMY AND RESEARCH IN HEALTH FIELD \\ Dr. Manuel Gutiérrez Henríquez \\ Unidad de Ergonomía. Facultad de Ciencias Biológicas. \\ UNIVERSIDAD DE CONCEPCIÓN
}

\begin{abstract}
T as investigaciones efectuadas en el sector salud indican que los principales riesgos _ocupacionales a los que están expuestos los trabajadores se relacionan con agentes biológicos, químicos, físicos, así como con factores de riesgo psicosociales y del ámbito de la ergonomía $(1,2)$. Al respecto, del punto de vista conceptual, la Ergonomía es una disciplina científica que estudia las interacciones entre seres humanos y sistemas, así como es la profesión que aplica teorías, principios, datos y métodos al diseño, con el objetivo de optimizar el bienestar del ser humano y el desempeño de los sistemas (3). Los temas que preferentemente son estudiados por la disciplina están relacionados con ergonomía física, cognitiva, organizacional y ambiental. Algunos ejemplos de tópicos específicos corresponden a trastornos músculo-esqueléticos relacionados con el trabajo, carga mental y diseño de sistemas de trabajo.
\end{abstract}

En el contexto de actividades laborales relacionadas con la atención en salud, si uno de los propósitos centrales de la ergonomía es optimizar la reacción entre bienestar humano y el desempeño de los sistemas, cabe la pregunta: ¿Qué investigaciones se deben desarrollar para alcanzar ese objetivo? En este sentido se puede sostener que se requiere efectuar estudios de ciencia básica, que permitan caracterizar a la población de trabajadores en términos de definir sus capacidades y limitaciones, su estado de salud y bienestar, así como establecer los determinantes asociados. También desarrollar ciencia aplicada que, sustentada en la identificación de los determinantes de salud y el conocimiento sobre capacidades y limitaciones de la población laboral, permita avanzar en estudios orientados a optimizar la relación entre personas y sistemas. De este modo, se deben responder interrogantes relacionadas con: ¿Qué problemas de salud presentan los trabajadores del sector salud? ¿Cuáles y qué importancia relativa tienen aquellos factores de riesgo que contravienen principios ergonómicos o dis-ergonómicos? ¿Cuáles son los mecanismos de acción de estos factores en la generación de daño en la salud? ¿Qué medidas son adecuadas para prevenir el daño? y ¿Qué estrategias de gestión son efectivas para optimizar la relación entre personas y sistemas de trabajo en el sector salud? 
En función de lo planteado, a continuación se describirán antecedentes de investigaciones desarrolladas en el contexto nacional, orientadas a responder estas interrogantes. Consideremos en primera instancia en el análisis: ¿Qué factores de riesgo dis-ergonómicos están presentes en actividades laborales de atención en salud? Para esbozar una repuesta se empleará como fuente de información la Encuesta Laboral (4), cuyo diseño es probabilístico y de cobertura nacional. Respecto de factores de riesgo biomecánicos relacionados con daño al sistema músculo-esquelético, para actividades económicas agrupadas en servicios sociales y de salud, la proporción de organizaciones con trabajadores expuestos a posturas incómodas es de un 18,6\%, para cargas pesadas un 15,7\% y movimientos repetitivos un 14,2 \% (4). En relación a la organización del trabajo, destaca la exposición a turnos nocturnos, estimándose que esta condición está presente en $23,7 \%$ de los trabajadores que se desempeñan en servicios sociales y de salud (4). En temas de ambiente físico, la proporción de organizaciones con trabajadores expuestos a ruido es de $10,7 \%$, a altas temperaturas un 8,4\%, vibraciones mecánicas un 2,4\% y falta de iluminación un 0,6\% (4). Respecto de la aplicación de encuestas de cobertura nacional, es relevante destacar que no se han publicado estudios que permitan tener una aproximación a la importancia relativa de factores de riesgo relacionados con carga mental y de demandas derivadas de la organización del trabajo en el sector salud. Tampoco se dispone de información que permita verificar tendencias a nivel nacional de los problemas de salud prevalentes de funcionarios de este sector económico. Esta carencia de información epidemiológica básica, obviamente limita la definición de prioridades de investigación y, más aún, de generación de conocimiento que apoye el desarrollo de políticas de salud pública y ocupacional para estas actividades laborales.

Respecto de estudios de cobertura más acotados, preferentemente en unidades hospitalarias, que tratan temas de ergonomía referidas al sector salud en Chile, las investigaciones incluyen contenidos relacionados a factores de riesgo de carga física y trastornos músculo-esqueléticos (TMEs), sistemas de turnos, carga mental y carga global de trabajo. En carga física se han estudiado labores relacionadas con el manejo de pacientes, en particular demandas biomecánicas de las tareas, así como prevalencia de trastornos músculo-esqueléticos y factores de riesgo asociados $(5,6)$. También, en servicios de atención de pacientes hospitalizados, unidades administrativas y laboratorio clínico, se han efectuado estudios de caracterización de factores de riesgo y de evaluación de medidas de prevención de TMEs (2). En temas de organización se han efectuado estudios de impacto sobre la salud, vida social y rendimiento del personal que efectúa cuarto turno (7). En carga mental se han caracterizado demandas percibidas por funcionarios de Unidades de Pacientes Críticos (8). Respecto de carga global de trabajo, se han desarrollado estudios en unidades de esterilización (9).

En cuanto a investigaciones dirigidas a establecer estrategias de prevención de daño en la salud de trabajadores del sector salud, los estudios efectuados han estado dirigidos preferentemente a la gestión de riesgos relacionados con TMEs (2). Las estrategias han incorporado fundamentos de gestión de riesgos laborales. En lo específico, para el desarrollo de diagnósticos y búsqueda de opciones de mejoramiento se han apli- 
cado enfoques de causalidad multifactorial y de gestión participativa (2). Al respecto, existen carencias relevantes de investigación aplicada en el sector salud, que genere conocimiento para apoyar la toma de decisiones en gestión de riesgos dis-ergonómicos relacionados con temas de carga mental y de tipo organizacional.

Otra línea de la cual existe carencia de investigación aplicada en el sector salud, dice relación con el propósito central de la ergonomía, referido a cómo generar condiciones de trabajo que optimicen el bienestar humano con el desempeño de los sistemas. Este es una línea de investigación donde la ergonomía basada en fundamentos de enfoques de análisis sistémicos puede efectuar aportes en innovación de productos y procesos de atención en salud. Este tipo de estudios requiere un vínculo muy estrecho y sostenido en el tiempo entre unidades de investigación y los equipos de los establecimientos de salud. Los modelos que se pueden aplicar para estas investigaciones requieren de una mirada interdisciplinaria y con objetivos que generen un desarrollo y beneficio mutuo entre las unidades de investigación y las organizaciones de salud. Un esquema que ilustra enfoques de esta naturaleza es el que se aprecia en la Figura 1. Como se representa, el modelo de gestión se aplica a organizaciones que tienen como compromiso brindar atención en salud y satisfacer requerimientos de bienestar de las personas. Para ello, la toma de decisiones debe basarse en el desarrollo de la investigación y el aprendizaje que surge de esos procesos. Como se puede apreciar, en la toma de decisiones del diseño y mejoramiento de los procesos de atención en salud, la investigación aborda los requerimientos y monitorea la retroalimentación de los usuarios internos y externos a la organización. En una mirada más amplia, en el marco de la responsabilidad social, se deben incluir los requerimientos del conjunto de contrapartes interesadas para las organizaciones.

Figura 1. Modelo de gestión organizacional en salud, que sustenta la toma de decisiones en procesos de investigación y aprendizaje, en función de los requerimientos de usuarios internos y externos.

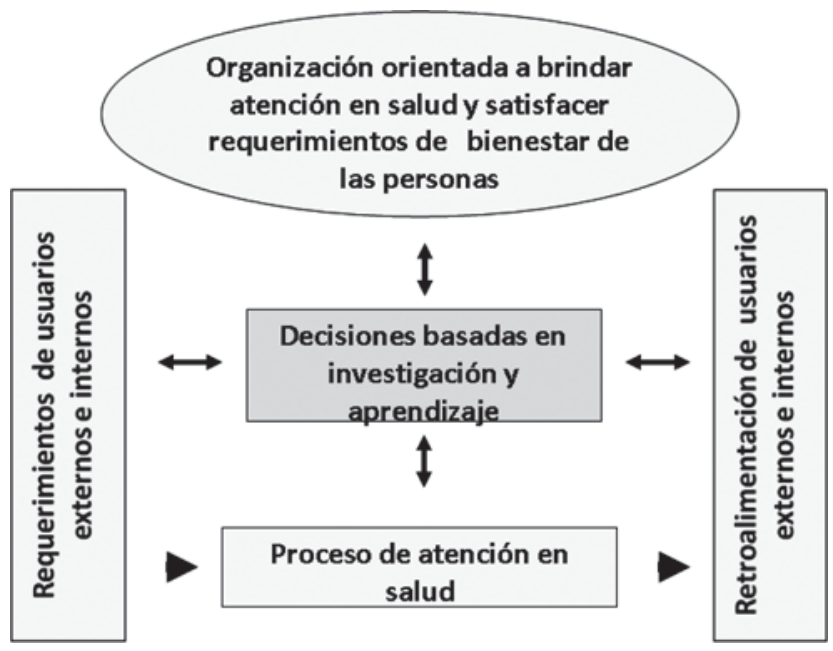


Como se puede concluir, existen diferentes brechas que abordar en temas de investigación en ergonomía a nivel de ciencia básica y aplicada en el sector salud. Estos requerimientos incluyen temas que van desde una adecuada caracterización de factores dis-ergonómicos, pasando por estudios que sustenten intervenciones y mejoramiento de condiciones de trabajo, hasta aportes en el desarrollo de aplicaciones y modelos de gestión que compatibilicen el bienestar humano y el desempeño de los sistemas de trabajo.

\section{REFERENCIAS}

1. Becker S, Carvalho M. Estudio sobre ausentismo en profesionales de enfermería del centro psiquiátrico de Manaus, Brasil. Rev. Latino-Am. Enfermagem. 2008; 16(1): 109-114.

2. Gutiérrez M, Monzó J, Lama O, Felmer A, Cruzat M, Bustos G. Ergonomía y gestión de riesgos de trastornos músculo-esqueléticos en unidades hospitalarias. Concepción: Universidad de Concepción; 2012.

3. International Ergonomics Association [Internet] [citado 21 Oct 2014]. Disponible en: http://www.iea.cc/whats/index.html.

4. Dirección del Trabajo. Séptima Encuesta Laboral 2011. Informe de resultados. Santiago de Chile: Dirección del Trabajo; 2011.

5. Pinto R, Córdova V, Silvestre R. Estudio de Caso: Comparación biomecánica entre métodos de transferencia en el Manejo Manual de Pacientes. Ponencia presentada en: VI Congreso Internacional de Prevención de Riesgos Laborales ORP; 14-16 mayo 2008; Coruña, España.

6. Gutiérrez M, Flores C, Monzó J. Prevalencia de Trastornos Músculo-Esqueléticos en Funcionarios de Centros Hospitalarios que Realizan Manejo de Pacientes y Caracterización de Potenciales Factores de Riesgo. Cienc Trab. 2010; 12(38): 447-453.

7. Córdova V, Hevia J, Figueroa A. Trabajo en turnos en el sector de la salud chileno: una comparación entre el sector público y privado. Cienc Trab. 2006; 8(21):147-150.

8. Ceballos P, Rolo G, Hernández E, Cabrera D, Paravic T, Burgos M. Factores psicosociales y carga mental de trabajo percibidos por profesionales de enfermería en Chile. Ponencia presentada en: Primer Congreso Nacional de Gestión Integrada; 9-11 julio 2014; Concepción, Chile.

9. Delgado D, Aguilera M, Delgado F, Cano I, Ramírez O. Calidad de Vida en el Trabajo y Condiciones de Trabajo, en Auxiliares de Esterilización. Cienc Trab. 2013; 15(48): 148-151. 